\title{
Sparse signal recovery in MIMO specular meteor radars with waveform diversity
}

\author{
Juan M. Urco, Jorge L. Chau, Tobias Weber, Senior Member, IEEE Juha Vierinen, and Ryan Volz
}

\begin{abstract}
Since the 1950s, specular meteor radars (SMRs) have been used to study the mesosphere and lower thermosphere (MLT) dynamic. Atmospheric parameters derived from SMRs are highly dependent on the number of detected meteors and the accuracy of the meteor's location. Recently, incoherent and coherent multiple-input-multiple-output (MIMO) radar approaches combined with waveform diversity have been proposed to increase the number of detected meteors and to improve time, altitude, and horizontal resolution. The incoherent MIMO approach refers to the addition of new transmit sites (widely separated), whereas the coherent MIMO refers to the addition of new transmit antennas in the same site (closely separated), in both cases, transmitting a different pseudorandom sequence from each antenna element. Unfortunately, the addition of new transmit antennas with different code sequences degrades the performance of conventional signal recovery algorithms. This is a consequence of the cross-interference between the transmitted signals, making it worse as the number of transmitters increase. In this work, we propose a signal recovery approach based on Compressed Sensing, taking advantage of the sparse nature of specular meteor echoes. The approach allows exact recovery of weak echoes even in interference environments. Besides the advantage of the proposed approach to recover the meteor signal, we discuss the optimal selection of the transmitted waveforms and the minimum code length required for exact recovery. Additionally, we propose a modification of the Orthogonal Matching Pursuit algorithm used in sparse problems to make it applicable in real-time analysis of large data. The success of the proposed approach is corroborated using Montecarlo simulations and real data from a multi-static spread spectrum meteor radar network installed in northern Germany.
\end{abstract}

Index Terms-Specular meteor radar, MIMO radar, spreadspectrum, waveform diversity, sparse recovery, compressed sensing, orthogonal matching pursuit, mesosphere and lower thermosphere.

\section{INTRODUCTION}

$\mathbf{M}$ ETEOROIDS entering the Earth's atmosphere heat up and ablate forming an ionized plasma trail. The plasma trails drift with the neutral wind. By the aid of radars, one can measure the trail velocity projected on the radar line-ofsight. Later, by combining several measurements, these are used to estimate the background wind. When the radar line-ofsight is approximately perpendicular to the trail, the scattered signal is strong and low-power radars can be used. This

J. M. Urco and J. L. Chau are with Leibniz-Institute of Atmospheric Physics at the Rostock University, Kühlungsborn, Germany (e-mail: urco@iapkborn.de, chau@iap-kborn.de)

T. Weber is with EE, University of Rostock, Germany (email:tobias.weber@uni-rostock.de)

J. P. Vierinen is with UiT, The Arctic University of Norway, Tromso, Norway (e-mail:juha-pekka.vierinen@uit.no)

R. Volz is with MIT Haystack Observatory, Massachusetts, USA (email:rvolz@mit.edu) perpendicular point is also known as the specular point. Since the 1950s, specular meteor radars (SMRs) have been used to characterize the atmospheric dynamic in the mesospheric and lower thermospheric (MLT) region [1]-[3].

Typically, mean wind estimations are done using several meteor detections within a certain volume and time, assuming horizontal homogeneity [4], [5]. The fidelity of the estimation is highly dependent on the number of meteor detections and the meteor location accuracy. Indeed, there are many thousands of meteors per minute entering to the Earth's atmosphere. However, only a few of them accomplish the specular condition and can be detected by a given SMR. Recently, multistatic meteor radar networks have been proposed to increase the number of meteor detections and to improve the time, altitude, and horizontal resolution of estimated wind fields [6]-[8]. A multi-static radar network consists of multiple transmitters (Txs) and multiple receivers (Rxs) placed in the same or at different locations. As described in [9], these radars can be classified as coherent and incoherent MIMO radars, respectively. Figure 1 shows a sketch of a coherent and an incoherent MIMO radar both with two transmitting and two receiving antennas. In case of a coherent MIMO, the $\mathrm{Tx}$ antennas are collocated or closely separated. Whereas in an incoherent MIMO the Tx antennas are widely separated. In order to separate the contribution of each transmitter some kind of diversity is required; either time, polarization, frequency or waveform diversity [9].

Recently, Stober and Chau [6] proposed a meteor radar network employing two transmit and multiple receive stations widely separated. The $\mathrm{Tx}$ stations work at two different frequencies and the $\mathrm{Rx}$ stations listen both frequencies with interferometry capability. This radar network can be classified as an incoherent MIMO radar using frequency diversity. Its main advantage is that this network can be implemented with commercial radars working at different frequencies, keeping the data analysis the same. Nevertheless, the complexity comes by using a broad spectrum bandwidth as the number of transmitters increases, complicating also the receiving side. Similarly, Vierinen et al. [7] proposed a multi-static radar network using Tx stations transmitting different pseudorandom code sequences at the same frequency, i.e. spread-spectrum, leaving the receive side unchanged. This network can also be classified as an incoherent MIMO radar but using waveform diversity. In the same way, Chau et al. [8] proposed the use of a combination of coherent and incoherent MIMO radars to simplify the deployment of these networks. Among other advantages, MIMO with waveform diversity allows reusing the spectrum bandwidth. However, it makes the decoupling 
of scattered signals coming from different transmitters more complicated.

In the past, the least squares estimator (LSE) has been used for signal recovery in MIMO communications systems for its simplicity and tolerable performance. The main limitation of LSE is that it can only be applied to overdetermined problems, which is not the case for multi-static SMR networks employing waveform diversity. In communications, LSE has mostly been applied to MIMO systems with more receivers than transmitters [10], [11]. In those works, the algorithm is called zero forcing linear estimator (ZFE), which yields the same solution as LSE.

In the multi-static SMR network proposed by [7], the maximum likelihood estimator (MLE) was used to find the same solution as the weighted least squares estimator (WLSE). Therefore, a trick was introduced to make the problem overdetermined. Recovering the meteor signal for each transmitter at a time and considering the contribution from other transmitters as noise. Since the pseudorandom codes are not fully orthogonal, this only works as long as the number of transmitters is small or when the transmitters are located far away from each other. Otherwise, the noise floor might increase considerably, hiding the weakest echoes under the cross-interference and reducing the number of detected meteors. Other approaches, like minimum mean square error (MMSE) [12], have been proposed to overcome this issue but at the price of increasing the computational power and requiring prior knowledge of the noise variance.

In this work, we introduce an approach based on compressed sensing (CS) to recover specular meteor echoes from radar measurements obtained in a multi-static SMR network, either from a coherent or an incoherent MIMO radar using waveform diversity. Unlike conventional techniques such as matched filter [13] or maximum likelihood [7], our approach is able to recover weak signals in interference environments.

Our paper is organized as follows. First we briefly describe MIMO radar model and some signal processing background. In section IV we describe the CS theory and how it is applied to MIMO SMRs. Then, we support our approach with simulations and experimental results. Finally we discuss the results and future improvements.

\section{SySTEM MODEL}

As explained in the introduction, winds are estimated by combining several meteor radial velocities with their corresponding location. The radial velocity can be obtained directly from one single receiving antenna, however, the meteor location has to be determined combining measurements from several antennas. This is known as radar interferometry [14]. The target backscatter coefficient at one receiving antenna can be defined as

$$
x_{i}(r)=a_{i}(r) \exp \left(-j \overrightarrow{k_{r}} \overrightarrow{d_{i}}\right)
$$

where $x_{i}(r)$ is the target echo at receiver $i$ for a given range $r, a_{i}(r) \in \mathbb{R}$ is the target coefficient amplitude, $\overrightarrow{k_{r}} \in \mathbb{R}^{3}$ is its Bragg vector, and $\vec{d}_{i} \in \mathbb{R}^{3}$ represents the receiving antenna coordinates. When the transmit waveform is coded [7], the signal model for a coded continuous wave SMR with one transmitter can be described by

$$
y_{i}=A x_{i}+\eta_{i}
$$

where $y_{i} \in \mathbb{C}^{M}$ is the measurement vector at the receiver " $i "$ and $x_{i}(r)$ is the unknown parameter vector, $x_{i} \in \mathbb{C}^{R}$ for simplicity. $A \epsilon \mathbb{C}^{M x R}$ is the sensing matrix and $\eta_{i} \sim N_{\mathbb{C}}(0, \Sigma)$ is the receiver noise with a Gaussian distribution, $\Sigma$ being the noise covariance matrix. The sensing matrix is a circulant matrix specified by the waveform vector $w \in \mathbb{C}^{M}$, where $M$ is the waveform length and $R$ is the number of range gates $(M \geq R)$.

$$
A=\left[\begin{array}{ccccc}
w_{[0]} & w_{[M-1]} & w_{[M-2]} & \ldots & w_{[M-R+1]} \\
w_{[1]} & w_{[0]} & w_{[M-1]} & \ldots & w_{[M-R+2]} \\
w_{[2]} & w_{[1]} & w_{[0]} & \ldots & w_{[M-R+3]} \\
\vdots & \vdots & \vdots & \ddots & \vdots \\
w_{[M-1]} & w_{[M-2]} & w_{[M-3]} & \ldots & w_{[M-R]}
\end{array}\right]
$$

Interferometry can be applied using signals received at different Rx antennas or signals coming from different $\mathrm{Tx}$ antennas. This is known as a MIMO radar [9]. In a meteor radar network with several transmitting antennas, i.e. a MIMO radar, the model becomes

$$
y_{i}=\sum_{j=1}^{t} A_{j} x_{i j}+\eta_{i}
$$

where $A_{j}$ is the sensing matrix due to the transmit waveform $w_{j}, x_{i j}$ is the unknown vector at receiver $i$ from transmitter $j, I$ is the number of receivers and $J$ is the number of transmitters using different waveforms. Equation 4 can be further simplified using

$$
y_{i}=\Phi X_{i}+\eta_{i}
$$

$$
\text { with } X_{i}=\left[\begin{array}{c}
x_{i 1} \\
x_{i 2} \\
\vdots \\
x_{i J}
\end{array}\right] \text { and } \Phi=\left[\begin{array}{llll}
A_{1} & A_{2} & \ldots & A_{t}
\end{array}\right]
$$

where $X_{i} \in \mathbb{C}^{N}, \Phi \in \mathbb{C}^{M x N}$, and $N$ is equal to the number of transmitters times the number of range gates $(N=J R)$.

In a MIMO radar with transmitting antennas widely separated, i.e. an incoherent MIMO (see Fig. 1), the signals $x_{i j}$ and $x_{i j^{\prime}}$ coming from transmitter $j$ and $j^{\prime}$ are incoherent since different transmit-receive links see different targets. On the contrary, when the transmit antennas are closely separated, i.e. a coherent MIMO radar, the signals $x_{i j}$ and $x_{i j^{\prime}}$ are coherent and the target echoes are located at the same range. Conventional algorithms treat the incoherent and coherent MIMO measurements the same way. However, in this work we will take advantage of the radar configuration to further improve the proposed algorithm. 


\section{SIGNAL PROCESSING BACKGROUND}

In the past, diverse approaches have been proposed to solve problems similar to (5). Among all of them, the matched filter estimator (MFE) [13] is the simplest and fastest algorithm, which is considered the optimal linear filter for maximizing the signal-to-noise ratio (SNR). MFE is recommended for detection of weak signals and its solution can be expressed as

$$
\hat{X}_{i}^{M F E}=\Phi^{H} y_{i}
$$

where.$^{H}$ is the Hermitian transpose operator and $\hat{X}_{i}^{M F E}$ is an estimation of $X_{i}$. When MFE is applied to a MIMO radar, it maximizes the SNR but also it enhances the sidelobes and the cross-interference between waveforms. MFE performance gets worse as the SNR and number of targets increase. Thus MFE is not applicable in MIMO systems and a different approach is required to reduce the interference. When $\Phi$ is full column rank, i.e. $M \geq J R$, the ordinary LSE can be applied to recover $X_{i}$ from (5). In this case, the problem has a unique LSE solution.

$$
\hat{X}_{i}^{L S E}=\underset{X_{i}}{\arg \min }\left\|y_{i}-\Phi X_{i}\right\|_{2}^{2}
$$

where the interference is reduced. The solution to (7) is given by

$$
\hat{X}_{i}^{L S E}=\Phi^{+} y_{i},
$$

where $\|.\|_{2}$ is the Euclidean norm and $\Phi^{+}=\left(\Phi^{H} \Phi\right)^{-1} \Phi^{H}$ is the Moore-Penrose pseudoinverse. Unfortunately, in most of the cases, $\Phi$ is not full column rank and there may be infinitely many LSE solutions of $X_{i}$ which fit $y_{i}$. For those cases, additional constraints are required to make the solution unique. A straight forward solution is to use the generalized inverse also called the truncated LSE [15], [16], where

$$
\begin{gathered}
\Phi^{+}=V S^{+} U^{T}, \\
S^{+}=\operatorname{diag}\left\{\tau_{k}\right\}, \tau_{k}=\left\{\begin{array}{ll}
1 / \delta_{k} & \text { if } \delta_{k}>0 \\
0 & \text { if } \delta_{k}=0
\end{array} .\right.
\end{gathered}
$$

$\Phi=U S V^{T}$ is the singular value decomposition, and $\delta_{k}$ is the $k$-th singular value. The main idea is to truncate the small singular values which might introduce high errors to the solution. In our case, the number of singular values greater than zero is equal to $\min (\mathrm{M}, \mathrm{N})$. In this way, even when the problem is underdetermined, we obtain a solution where the interference is reduced. In other disciplines, the truncated LSE is also known as the min-norm estimator, given that it minimizes the total energy in $X_{i}$. This is not convenient when recovering weak signals but it is good to reduce the cross-interference between waveforms. In order to find a balance between MFE and the truncated LSE, an additional regularization term is added to (7):

$$
\hat{X}_{i}^{R L S E}=\underset{X_{i}}{\arg \min }\left\|y_{i}-\Phi X_{i}\right\|_{2}^{2}+\lambda\left\|X_{i}\right\|_{2}^{2}
$$

The solution to (10) is given by

$$
\hat{X}_{i}^{R L S E}=\left(\Phi^{H} \Phi+\lambda I\right)^{-1} \Phi^{H} y_{i} .
$$

Equation 11 is known as Regularized Least Squares estimator (RLSE) or Ridge regression [17]. Where $\lambda>0$ represents the trade-off between the minimization of the residual and the total energy in $X_{i}$. Notice that, when $\lambda=0$, the RLSE solution is the same as the ordinary LSE, minimizing the total energy in $X_{i}$. On the other hand, when $\lambda$ tends to infinite, RLSE has a similar solution as MFE, maximizing the energy in $X_{i}$. A crucial point in RLSE is the selection of the regularization parameter $\lambda$, which can be found using crossvalidation algorithms [18], [19]. Unfortunately, this additional step increases the computational cost of the algorithm and not always the selected $\lambda$ is the best. Similar results can be found using the minimum mean square error estimator (MMSE), for which $\lambda=\frac{1}{S N R}$ [20].

Even using RLSE or MMSE the cross-interference is not significantly reduced if the matrix $\Phi$ is ill-conditioned. In MIMO communication channels, Successive Interference Cancellation (SIC) approaches have been employed to improve further the performance of linear detectors like ZF or MMSE [12], [21], [22]. These algorithms can be related to the CLEAN algorithm used in radio astronomy [23], where a set of point sources which describes the data well are found through successive point source cancellation. CLEAN is considered by several authors as the most basic implementation of the Orthogonal Matching Pursuit algorithm [24], which is used in CS to solve sparse problems.

\section{Compressed Sensing}

Compressed sensing (CS), also known as sparse recovery [25], [26], is an emerging theory which aims to solve undetermined problems like (5). Traditionally, the Nyquist theorem held that an arbitrary signal has to be sampled at twice its bandwidth for exact recovery. In contrast, CS claims that a signal can be recovered even from a very limited number of measurements if two conditions are fullfilled: (a) the signal is $K$-sparse in some domain, i.e., the number of non-zero values is less than $K$; (b) the sensing matrix satisfies the Restricted Isometry Property (RIP) [27], which requires that any $K$ columns of $\Phi$ are approximately orthogonal. Even though this may seem impossible, numerous authors have proven the robustness and efficacy of CS even when the signal is approximately sparse and noisy [26]-[30].

In case of a MIMO radar using waveform diversity, both conditions are satisfied. First, the meteor echoes can be considered as point targets and only a few of them are observed at a given range and time. Thus most of the values in $X_{i}$ are zeros. Indeed meteor echoes are sparse in space, which includes sparsity in range and angle. However, in this work we have only exploited the sparsity in range. Secondly, by selecting the proper waveforms the matrix $\Phi$ satisfies the RIP condition. Further details how to select the waveforms are described in section IV-B. For now we assume this is the case.

Similar to RLSE, CS adds a constraint to (5) to recover the sparsest solution under the two conditions described above: 


$$
\begin{gathered}
\hat{X}_{i}{ }^{C S}=\underset{X_{i}}{\arg \min }\left\|y_{i}-\Phi X_{i}\right\|_{2}^{2}, \\
\text { subject to }\left\|X_{i}\right\|_{0}<K,
\end{gathered}
$$

where $\left\|X_{i}\right\|_{0}$ is the $l_{0}$-norm, which counts the number of non-zero values in $X_{i}$. Equation 12 recovers the solution $\hat{X}_{i}{ }^{C S}$ with the smallest possible number of non-zeros which fit with the data $y_{i}$. Unfortunately, this problem is known to be NPhard and computationally intractable, i.e., its computational complexity can be exponential or factorial. Noticeably, [25], [26] show that the problem in (12) can be relaxed and reformulated using the $l_{1}$-norm, making it more computationally attractive. This is also known as Basis Pursuit (BP). Even though fast algorithms have been introduced to solve the BP problem and to reduce its complexity to $O\left(N^{4} M K\right)$ [31], this is still not applicable when facing real-time analysis for large data.

Greedy algorithms have been shown to be practical and efficient to solve (12). Note that, greedy algorithms are those that look for the locally optimal solution at each step with the intent to find the global optimum [32], [33]. Recently, the reduced complexity of the well-known Orthogonal Matching Pursuit (OMP) algorithm [24] and its variations [34]-[37] have attracted great attention for its simplicity and performance. Although OMP requires more measurements than BP both have the same accuracy, the low computational cost of OMP makes it feasible for real-time applications.

\section{A. Sparse recovery}

OMP is an iterative greedy algorithm which at each iteration selects the column of $\Phi$ which maximizes the correlation with the residual, then the index of this column is added to a list of selected columns, finally the contribution of the columns are eliminated from the measurements, generating a new residual for the next iteration. Even though OMP has a low complexity $O\left(2 N M K+3 M K^{2}\right)$ [35], some studies have proposed some modifications to improve its computational efficiency and recovery performance. For example in [35] the Generalized Orthogonal Matching Pursuit (GOMP) method is described, where more than one indices are identified in each iteration. GOMP reduces the algorithm complexity to $O(2 N M k)$, being $k$ the number of iterations. Similarly, in [34] the Stagewise Orthogonal Matching Pursuit (StOMP) is proposed, where indices higher than a threshold are selected in each iteration. In this work we employ a variation of StOMP since it does not require prior knowledge of the sparsity. The StOMP algorithm is described in Table I.

In StOMP, the selection of the threshold $t$ and the number of stages $s$ are directly related, and they both depend on the orthogonality of $\Phi$ and the sparsity of $X_{i}$. In order to reduce the number of stages, a small threshold can be chosen. However, as a consequence, the number of false detections (false non-zero values) might increase. This is mainly due to the fact that the MFE is used in the first step of StOMP. As discussed before, the MFE does not suppress well the sidelobes due to the high non-zero values which might be confused as real
TABLE I

\begin{tabular}{|c|c|}
\hline Input & $\begin{array}{l}\text { measurements at receiver } i, y_{i} \epsilon \mathbb{C}^{M} \\
\text { sensing matrix } \Phi \in \mathbb{C}^{M x N} \\
\text { threshold } t \in \mathbb{R} \\
\text { number of stages } s \in \mathbb{N}\end{array}$ \\
\hline Inititalize & $\begin{array}{l}\text { iteration count } k=0 \\
\text { residual vector } r^{0}=y_{i} \\
\text { support } S^{0}=\emptyset\end{array}$ \\
\hline \multicolumn{2}{|l|}{ Iteration } \\
\hline While $(k<s)$ & $\begin{array}{l}\text { Choose the columns } \Phi_{r} \text { of } \Phi \text { which satisfies } \\
R_{s}=\left\{r:\left|\hat{X}^{k}(r)\right|^{2}>t \sigma_{n}^{2}\right\} \\
\text { where } \hat{X^{k}}=\Phi^{H} r^{k-1} \\
\text { and } \sigma_{n}^{2} \text { is the noise variance in } \hat{X}^{k} \text {. } \\
\text { Merge the new subset with the previous support } \\
S^{k}=S^{k-1} \cup R_{s} \\
\text { Compute a better approximation of } X^{k} \text { supported by } S^{k} \\
\hat{X}^{k}=\left(\Phi_{S^{k}}^{H} \Phi_{S^{k}}^{H}\right)^{-1} \Phi_{S^{k}}^{H} y_{i} \\
\text { Update the residual for the next iteration } \\
r^{k}=y_{i}-\Phi_{S^{k}}^{H} \hat{X}^{k} \\
\mathrm{k}=\mathrm{k}+1\end{array}$ \\
\hline Output & the estimated signal at receiver $i, \hat{X}_{i}=\hat{X}^{k}$ \\
\hline
\end{tabular}

STOMP ALGORITHM

echoes. Consequently, we propose a modification of StOMP, employing the truncated LSE in the first iteration instead of MFE. The truncated LSE allows us to identify correctly the highest non-zero values in the first iteration which finally introduce the highest errors. Then we employ the MFE to maximize the small non-zero values, making them detectable in the next step. The modified StOMP is resumed in Table II and it allows to recover the meteor signal using only two stages, for which we named it as Fast Stagewise Orthogonal Matching Pursuit (FaStOMP).

FaStOMP helps to identify the high non-zero values in $X_{i}$ and reduce the interference between waveforms. However, we noticed that the non-zero values close to the noise level were not recovered even using FaStOMP. To help the recovery of weak echoes, we employed a technique similar to the Joint and Block Sparsity used in [38], [39]. Basically, it modifies the identification step in StOMP and FaStOMP, selecting the columns where the coherently integrated values along the time are higher than the selected threshold, i.e.

$$
\hat{X}(r)=\frac{1}{L} \sum_{t=-L / 2}^{L / 2} \hat{X}(r, t),
$$

this is possible given that the meteor echoes should last for at least few ms (L samples). Similarly, the signal can be incoherently integrated along the Rx and Tx channels to improve the detectability, i.e.

$$
\left|\hat{x}_{i j}(r)\right|^{2}=|\hat{x}(r)|^{2}=\frac{1}{I J} \sum_{i=1}^{I} \sum_{j=1}^{J}\left|\hat{x}_{i j}(r)\right|^{2} .
$$

Hereafter, when we refer StOMP or FaStOMP, they both include the coherent and incoherent integrations just described. 
TABLE II

FASTOMP ALGORITHM

\begin{tabular}{ll}
\hline Input & $\begin{array}{l}\text { measurements at receiver } i, y_{i} \epsilon \mathbb{C}^{M} \\
\text { sensing matrix } \Phi \in \mathbb{C}^{M x N} \\
\text { threshold } t \in \mathbb{R} \\
\text { number of stages } s \in \mathbb{N}\end{array}$ \\
\hline Inititalize & iteration count $k=0$ \\
& residual vector $r^{0}=y_{i}$ \\
& support $S^{0}=\emptyset$
\end{tabular}

A diagram of the algorithm used to recover specular meteors from MIMO radar measurements is shown in Fig. 2.

\section{B. Sensing matrix design}

Exact recovery of a $K$-sparse vector $X_{i}$ requires the sensing matrix $\Phi$ to satisfy certain conditions. In the literature, diverse approaches have been proposed to guarantee exact recovery: the RIP [27], the uniform uncertainty principle (UPP) [40], the exact recovery condition (ERC) [32], and the mutual incoherence condition (MIC). Except for MIC and ERC, the rest of them are not feasible to apply in practice. In order to optimize the design of the sensing matrix, we make use of mutual coherence introduced in [41]:

$$
\mu_{\text {max }}=\max _{p \neq q}\left|\Phi_{p}^{H} \Phi_{q}\right|,
$$

where $\Phi_{p}$ is the $p$-th column of $\Phi$ and $\mu_{\max }$ represents the largest off-diagonal element of the gram matrix $G=\Phi^{H} \Phi$. In some sense, MIC measures how orthogonal are the columns in $\Phi$. According to [32], in the worst-case scenario, the sparsity $K$ is bounded by the mutual coherence

$$
K_{\max }<\frac{1}{2}\left(1+\frac{1}{\mu_{\max }}\right),
$$

This means that a signal $X_{i}$ with more than $K_{\max }$ non-zero elements cannot be recovered for a given $\Phi$. For a more relaxed scenario, other authors suggest to use an average value of the mutual coherence $\mu_{\text {avg }}$. This is a more adequate scenario in our problem given that the distribution of the non-zero values (meteors) are equally probable along $X_{i}$. Finally, the optimal sensing matrix is the matrix $\Phi$ which minimizes $\mu_{\text {avg }}$.

As described in section II, the matrix $\Phi$ is a function of the waveforms $w_{j}$. In order to optimize $\Phi$, we have to select a proper set of waveforms $w_{j}$ which minimize $\mu_{\text {avg }}$. Similar to [7], we employ pseudorandom binary phase-coded sequences as waveforms. Although they are not fully orthogonal, they maximize the transmit energy, are easy to be generated, and have good orthogonality properties [42]. Similarly, other authors suggest the use of quasi-orthogonal codes [43], [44]. In our case, the waveform amplitude is a constant equal to 1 , i.e., $\left|w_{j[m]}=1\right|$, and the phase can only be 0 or $\pi$. The phase sequence for each waveform is selected randomly from a given seed number. Thus, the problem reduces to a selection of a number of seeds equal to the number of transmitters which minimizes $\mu_{\text {avg }}$.

Further optimization can be done in case a coherent MIMO radar is employed. As described, the MIC condition measures how large the off-diagonal elements of the gram matrix $G$ are, which can be seen as a measure of how strong the crossinterference between two columns of $\Phi$ is, where a column of $\Phi$ represents a shifted version $w_{j}(\tau)$ of the waveform $w_{j}$. In general, we are interested in minimizing every possible combination of $\left(w_{j}\left(\tau_{1}\right), w_{j^{\prime}}\left(\tau_{2}\right)\right)$ for $j \neq j^{\prime}$, given that they are equally probable. This is not the case in a coherent MIMO radar. When a target echo is detected at a given range in one Tx channel, i.e., $x_{i 1}(\tau) \neq 0$, it also appear at the same range in the other Tx channels, i.e. $x_{i j}(\tau) \neq 0$ for $j=[2, \ldots J]$. Hence the probability of having the combination $\left(w_{j}(\tau), w_{j^{\prime}}(\tau)\right)$ is $100 \%$, which must be further minimized. The interference between waveforms at lag $\tau$ is the same as at lag 0 and it can defined as $\mu_{j j^{\prime}}=w_{j}^{H} w_{j^{\prime}}$. For the experiments and simulations presented in this work, we have used $\mu_{a v g}$ and $\mu_{j j^{\prime}}$ as conditions to select the set of waveforms, i.e. the different seeds.

The value of the mutual coherence $\mu_{a v g}$ was selected using (16) based on the maximum number of meteor echoes expected in a SMR. Considering a MIMO radar with 5 transmitters and an average of 5 echoes per unit time we get $K=25$ and $\mu_{a v g}=2 e^{-2}$. Similarly, we selected $\mu_{j j^{\prime}}=2 e^{-3}$. Once the two parameters are defined we use a exhaustive or iterative search to find the proper seeds which accomplish the conditions.

\section{RESULTS}

Preliminary results using the proposed approach in a multistatic meteor radar system deployed in northern Germany have been published in [8]. The network consisted of one Tx station with five transmitting antennas and one receiving station with five receiving antennas, i.e., a MIMO link. Given that CS is defined based on probabilities and unstable conditions, it is very difficult to prove the success of the approach only based on one campaign. Thus we conducted simulations to support our proposal and define the limits of this. The simulated network is similar to the MIMO link used in [8].

\section{A. Simulations}

Here, we consider a coherent MIMO radar consisting of five transmitting and five receiving antennas, where each Tx antenna transmits a different waveform. The waveforms were optimal selected using the conditions described in section IV-B, resulting in the following seeds $=[1,97,173,1885,8928]$. 
In order to evaluate the performance of the recovery algorithms, we conducted a Montecarlo simulation with 500 runs using (5) under three different scenarios (a) variable SNR (b) variable sparsity and (c) variable waveform length. In the three cases, the noise variance $\sigma_{n}^{2}$ was set to 1 and the signal power of the simulated meteors $\sigma_{s}^{2}$ was relative to the noise $\left(S N R=\sigma_{s}^{2} / \sigma_{n}^{2}\right)$. Given that we are simulating a coherent MIMO radar, the meteor range gates were randomly selected but they were kept the same along the Rx and Tx channels, i.e. $\left|x_{i j}(r)\right|=\left|x_{i j^{\prime}}(r)\right|$ for $j, j^{\prime}=[1, \ldots, J]$.

In our simulations, once $X_{i}$ was defined, ergo $y_{i}$ was known, so we could estimate $\hat{X}_{i}$ from $y_{i}$ using different algorithms. In all the cases, the sparsity $K$ defined as the number of simulated meteors times the number of transmitters was only used to corroborate the success of the recovery algorithms.

Figure 3 shows a comparison of the recovery error as a function of SNR for three algorithms (a) MFE, (b) truncated LSE, (c) StOMP. For simplicity, only two meteor echoes, i.e. $\mathrm{K}=10$, were considered in this simulation. Figure 3(a) shows the results when the two meteor echoes have the same SNR but they are located randomly in range. Clearly, under these conditions, most of the algorithms work properly when the SNR is greater than $-15 \mathrm{~dB}$. Therefore, it is clear that MFE has a good performance for low SNR targets. However, StOMP improves even further the recovery of weak signals. This is due to the ability of our StOMP implementation to reduce the noise rms level after integrating the signal. When no integration is used in the StOMP implementation, MFE and StOMP have similar performance. FaStOMP was not included in this case given that it had the same performance as StOMP for these conditions. Figure 3(b) shows a similar simulation than Fig 3(a) but for which the two meteors have a SNR difference. One of them was fixed to $-5 \mathrm{~dB}$ and the other one fluctuated from $-5 \mathrm{~dB}$ to $30 \mathrm{~dB}$. As expected, we observe that as soon as the SNR difference is larger than $10 \mathrm{~dB}$, the truncated LSE has a better performance than MFE. This indicates that when two signals coexist within $X_{i}$ and they have a high SNR difference the truncated LSE is able to minimize the interference between transmitted signals.

The relative error used in the plots was defined as

$$
\Delta e=\sum_{k=1}^{k=K} \frac{\left|X_{i}(k)-\hat{X}_{i}(k)\right|^{2}}{\left|X_{i}(k)\right|^{2}},
$$

where $X_{i}(k)$ is the k-th non-zero value of $X_{i}$. We decide not to use the typical mean-square-error given that this is always minimized by CS. Instead, we weighted it by the power of $X_{i}(k)$ since we are interested in the total error independent of the signal magnitude. The final error is averaged along the 500 runs. Since we are not taking into account the error coming from the zero elements, we have added to the plots the number of non-zero elements in $\hat{X}_{i}$ for which the power is higher than a threshold. Being conservative we have selected a threshold of $5 \sigma^{2}$, where $\sigma^{2}$ is the estimated variance from the decoded data.

Another important question in recovery problems is: how sparse our problem is, i.e., what is the maximum $K$ for which exact recovery is guaranteed? According to (16) for the selected waveforms, the maximum $K$ is 26 . Figure 4 shows the performance of the algorithms as a function of sparsity for (a) MFE, (b) truncated LSE, (c) StOMP, and (d) FaStOMP. For this simulation, the range location of the meteors and the SNR was randomly selected. Clearly, MFE and the truncated LSE starts to fail when $K$ is greater than 50. The number of false echoes were not included for MFE and LSE given that they were too high. On the other hand, the CS results prove that the proposed method works successfully, even when the sparsity is about 250. When the sparsity is less than 200 there is no difference between StOMP and FaStOMP. If we consider a relative error of $5 \%$ as acceptable, the maximum sparsity for StOMP and FaStOMP are 255 and 340, respectively. The improvement from StOMP to FaStOMP proves that using LSE instead of MFE in the first OMP iteration helps a lot to properly select the highest non-zero values, which finally introduce the highest errors. The number of false echoes for both cases are below 5, which is a good indicator that the algorithms are working correctly.

A similar analysis was done to test the minimum code length required for exact recovery. Here we assume a maximum number of meteors at a given time equal to 20 and a maximum number of ranges equal to 300. The results are shown in Fig. 5. Using the same relative error of 5\%, the sparsity for StOMP and FaStOMP are 395 and 350, respectively, meaning that we can recover exactly up to 20 meteors in a MIMO radar with 5 Txs and 5 Rxs, even when using a short waveform, i.e., for a typical SMR network a waveform length of 400 bits would be good enough.

\section{B. Multi-static specular meteor radar campaign}

In addition, we present the results from a multi-static spread-spectrum meteor radar system deployed in northern Germany. Similar to our simulations, the system consists of one Tx station with five antennas located in Kühlungsborn, Germany $\left(54.11^{\circ} \mathrm{E}, 11.76^{\circ} \mathrm{N}\right)$ and one $\mathrm{Rx}$ station with five antennas located in Neutrelitz, Germany $\left(53.33^{\circ} \mathrm{E}, 13.07^{\circ} \mathrm{N}\right)$. Each antenna transmitted a continuous waveform with a transmit power of $400 \mathrm{~W}$. The waveform length and the number of range gates used were 1000 and 350, respectively. Further details of this network can be found in [8].

Figure 6 shows the range time intensity (RTI) plot of specular meteor echoes after applying three different algorithms to recover the meteor signal (a) MFE, (b) truncated LSE, and (c) FaStOMP. As expected, MFE allows to detect weak echoes but it fails in the presence of strong airplane or meteor echoes, given that OMP enhances the sidelobes and cross-interference between waveforms. This does not allow to distinguish between real echoes and artifacts. On the other hand, the truncated LSE reduces the cross-interference for the strong echoes but as a consequence, the weak echoes are missing. Therefore, in case of very strong echoes, we can still see some sidelobes. This is due to the sensing matrix is ill-conditioned. Finally, Fig 6(c) shows the results by using FaStOMP. In this case, the strong and weak echoes were perfectly recovered, and the sidelobes were eliminated. Since the FaStOMP approach only recovers the non-zero values, the 
noise level was estimated from the residual and added to the data synthetically.

\section{DISCUSSION AND CONCLUDING REMARKS}

This work introduces a new approach based on compressed sensing (CS) to recover meteor signal echoes from a MIMO radar network using waveform diversity. In a MIMO radar, the recovery problem becomes undetermined and some regularization is required to retrieve the meteor signals. Common approaches like MFE or LSE do not decouple well the signals coming from different transmitters. The approach proposed here takes advantage of the sparse nature of the meteor echoes to regularize the problem by choosing the sparsest solution which matches the measurements.

The algorithm selected to find the sparsest solution was a variation of the Orthogonal Matching Pursuit algorithm (OMP) given its great performance and simplicity, which is more recommended than its counterpart Basis Pursuit (BP). Even though it is known that OMP requires a larger number of measurements than BP to recover a signal with the same accuracy, its computational cost makes it much more attractive for real-time applications.

Including the truncated LSE to OMP, i.e. FaStOMP, we could recover the meteor signal from the radar measurements using two iterations only. The reduced complexity of the proposed sparse recovery approach makes it applicable even for large data sets. In order to have a rough idea of the computational time we tested our implementation in two different scenarios: (a) a MIMO link consisting of 5 Tx channels and 1 Rx channel, and (b) a MIMO link consisting of 5 Tx channels and $5 \mathrm{Rx}$ channels, i.e. 25 channels in total. For the first case we used a Core i5 PC with 8GB of RAM to process the MIMO radar data, for which the processing time was around $2 \mathrm{~s}$ for $60 \mathrm{~s}$ of data. In the second case we used a Core i7 PC with 16GB of RAM, getting $12 \mathrm{~s}$ to process $60 \mathrm{~s}$ of data. In both cases the radar data was acquired continuously at a sample rate of $100 \mathrm{kHz}$ and the algorithm was implemented in Python using the Intel's optimized version of numpy and scipy libraries [45]. Even though the new python libraries allow us to use more than one core, our implementation made use only of one core at a time.

Aditionally, the CS approach can help to reduce or compress the meteor radar data. Since FaStOMP only recovers echoes above a threshold, most of the data contains zeros and can be compressed using conventional compression filters. In our application we used the HDF5 file format with gzip compression to save the decoded data. By doing this, the hard drive space required for decoded data was reduced almost 60 times compared with data obtained applying conventional algorithms. Furthermore, standard meteor analysis involves decoding and meteor detection, however, by employing CS we could join the two steps in one.

In this work, we postulate the recovery problem assuming the presence of specular meteors only. However, distinct atmospheric targets like non-specular meteors, E-region irregularities, airplanes, ground clutter, etc. might be included in the radar data. In those cases, the echo is not concentrated in

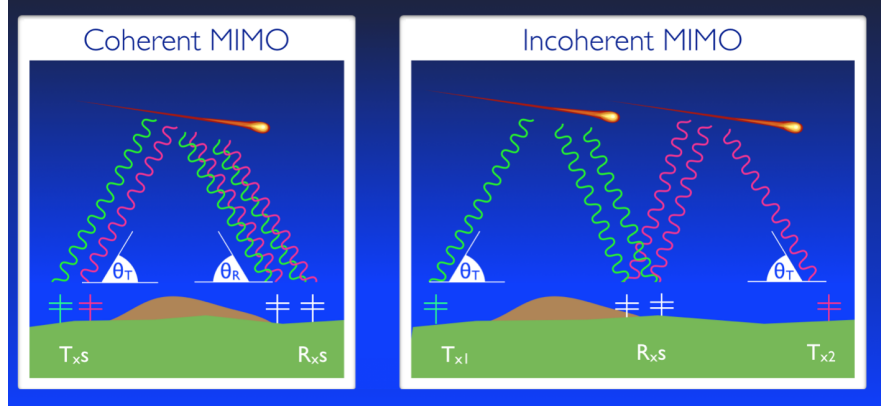

Fig. 1. A sketch representing two kind of meteor radar networks: (a) a coherent MIMO radar (b) an incoherent MIMO radar. Only one receiver station is drawn for simplicity.

one range only and, instead, it might be spread in several ranges. As the simulations show, even in those cases, the recovery algorithm works successfully as long as the number of non-zero values is less than one-third of the number of measurements. Figure 7 shows an example of a strong nonspecular meteor echo detected by the proposed algorithm where the target is spread along 50 ranges, perhaps associated to a fireball. A transform domain, where these non-pointtargets are more sparse can be added to (5) in the future to further improve the recovery of these type of echoes.

Another future task is to additionally consider the waveform errors in the problem. Along with this work, we have considered an ideal transmitted waveform, which is not the case in the real world. The synthesized signal passes through bandwidth limited filters, amplifiers, and antennas before being irradiated. This introduces imperfections to the transmitted signal which were not taken into account in our model, i.e., the matrix $\Phi$ in (5). It could be problematic in the presence of high SNR echoes given that the imperfections might also be high. We experienced this problem in a quasi-monostatic link where the transmitter and the receiver station were only $5 \mathrm{~km}$ apart, observing a strong ground clutter with an SNR of $60 \mathrm{~dB}$. In the future, errors in the matrix $\Phi$ can be considered in the equation and the total least squares estimator algorithm [46] can be applied instead of the ordinary LSE in our FaStOMP implementation.

Finally, as suggested by [8], the proposed approach can be extended to a network formed by pulse radars using a relatively long coded pulses combined with time diversity. As it was done in [9]. Even though the cross-interference between waveforms is higher when pulse radars are used, the problem might be still solvable if the signal is sparse enough.

\section{ACKNOWLEDGMENT}

This work was partially supported by the Deutsche Forschunggemeinschaft (DFG, German Research Foundation) under SPP 1788 (CoSIP)-CH1482/3-1 and by the WATILA Project (SAW-2015-IAP-1). The authors gratefully acknowledge the support of the international team by the International Space Science Institute (Bern, Switzerland) and discussions within the ISSI Team 410. Some hardware, software, and analysis work at MIT Haystack Observatory was supported by 


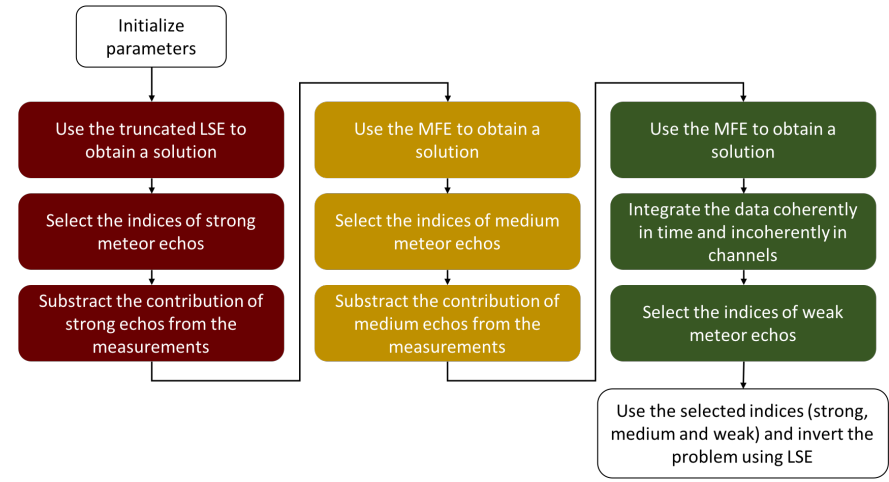

Fig. 2. A resumed flow chart describing the sparse algorithm used to recover specular meteor echoes. The red, yellow and green boxes represent the estimation and detection of the strong, medium and weak meteor echoes, respectively.
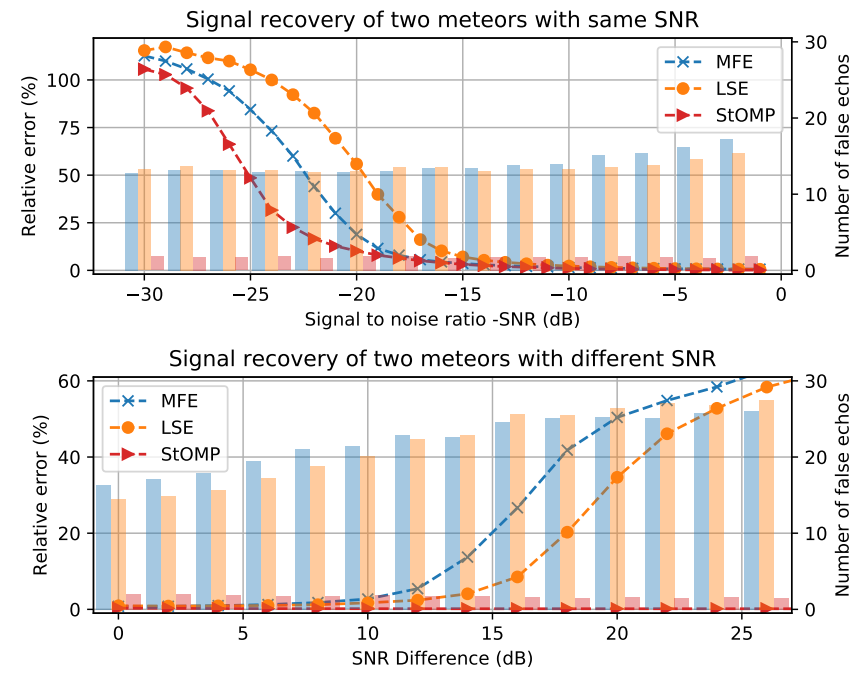

Fig. 3. Performance comparison of recovery techniques as a function of SNR. Every simulation run contain two meteor targets with (a) both having the same SNR and (b) having a SNR difference indicated in the plot, with the weakest one fixed to $-5 \mathrm{~dB}$. The colored bars indicate the average number of false echoes detected by each algorithm. During the simulation $M, N$, and $K$ were set to 1000,2000 , and 10 respectively (see text for details).

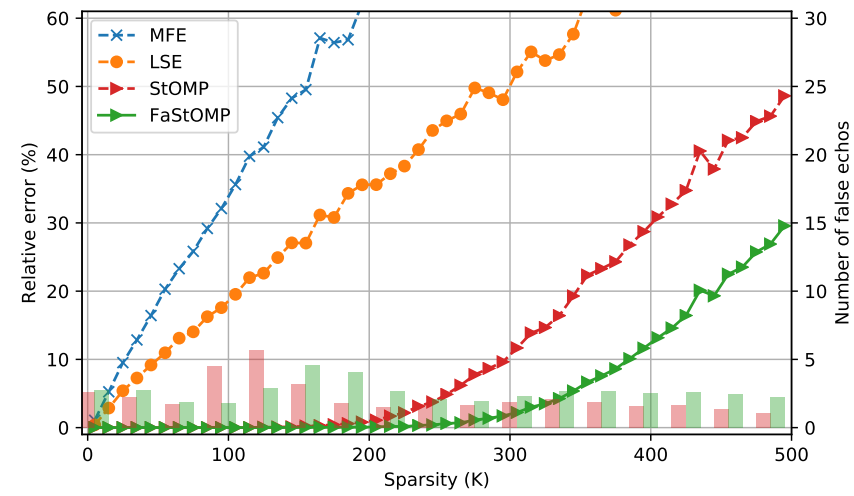

Fig. 4. Same as Fig. 3. Recovery performance as a function of sparsity. In every simulation run, the simulated meteors had random SNR and were randomly located in range. Only colored bars for the last two techniques are included. $M, N$, and $\mathrm{SNR}_{\max }$ were set to 1000,2000 , and 25 respectively.

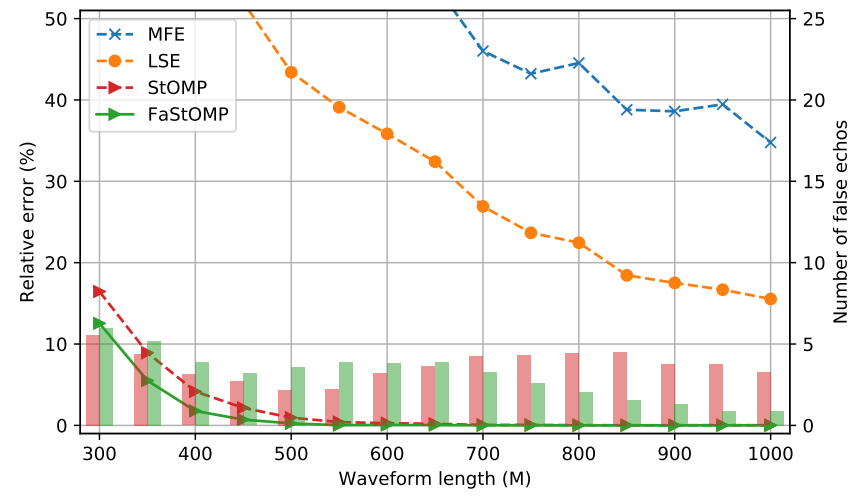

Fig. 5. Same as Fig. 4. Recovery performance as a function of waveform length. $N, K$, and $\mathrm{SNR}_{\max }$ were set to 1500,100 , and 25 respectively.
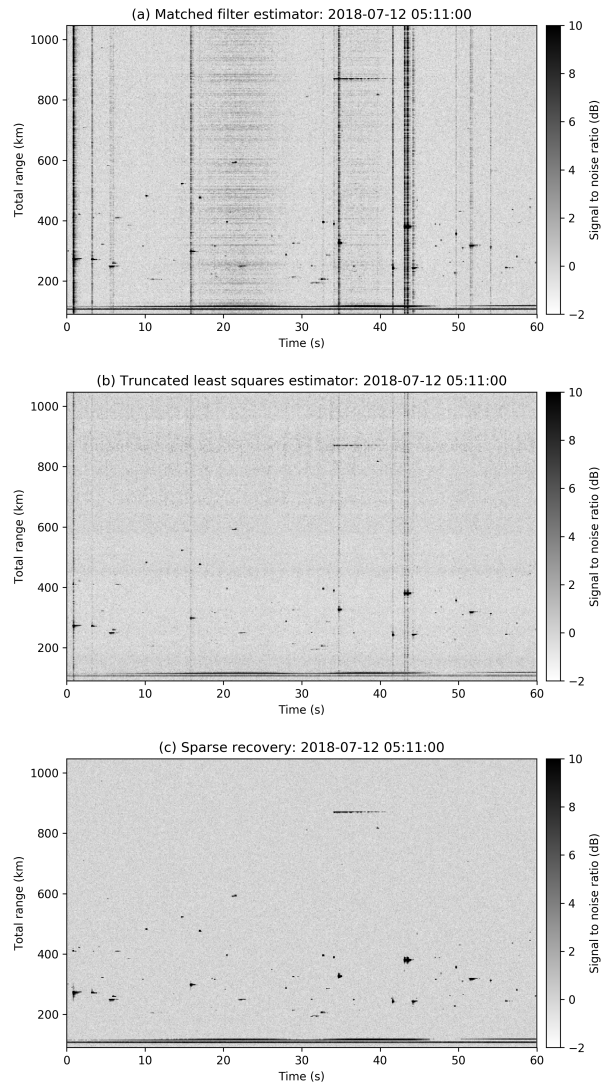

Fig. 6. Performance comparison of recovery techniques with data acquired in a meteor radar network in northern Germany. (a) Matched filter estimator (b) Least squares estimator (c) Sparse recovery using FaStOMP 


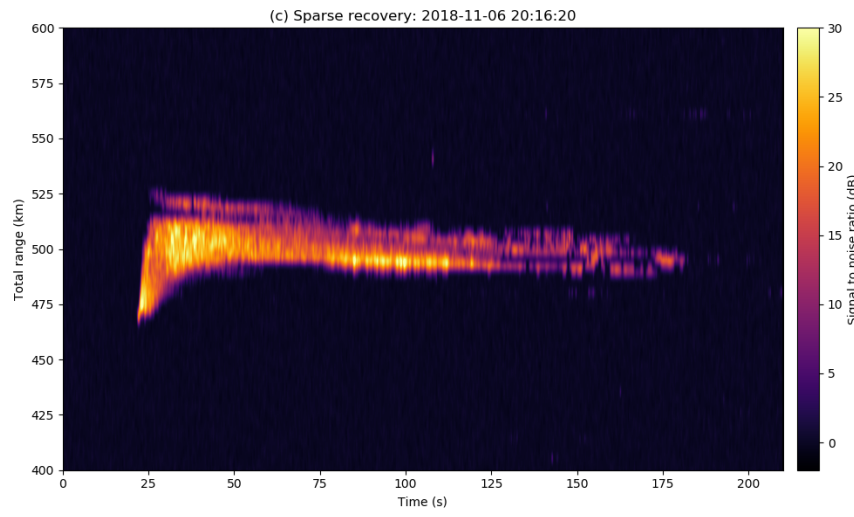

Fig. 7. An example of a target spread along several ranges recovered by the sparse approach.

NSF Major Research Infrastructure grant AGS-1626041. We also thank Kiara Chau for sketching our proposed systems in Figure 1.

\section{REFERENCES}

[1] L. A. Manning, O. G. Villard, and A. M. Peterson, "Meteoric echo study of upper atmosphere winds," Proceedings of the IRE, vol. 38, no. 8, pp. 877-883, Aug 1950.

[2] D. S. Robertson, D. T. Liddy, and W. G. Elford, "Measurements of winds in the upper atmosphere by means of drifting meteor trails I," Journal of Atmospheric and Terrestrial Physics, vol. 4, p. 255, 1953.

[3] W. Elford and D. Robertson, "Measurements of winds in the upper atmosphere by means of drifting meteor trails II," Journal of Atmospheric and Terrestrial Physics, vol. 4, no. 4, pp. 271-284, 1953.

[4] W. K. Hocking, B. Fuller, and B. Vandepeer, "Real-time determination of meteor-related parameters utilizing modern digital technology," Journal of Atmospheric and Solar-Terrestrial Physics, vol. 63, pp. 155-169, Jan. 2001.

[5] D. A. Holdsworth, I. M. Reid, and M. A. Cervera, "Buckland park all-sky interferometric meteor radar," $R a$ dio Science, vol. 39, no. 5, 2004. [Online]. Available: https://agupubs.onlinelibrary.wiley.com/doi/abs/10.1029/2003RS003014

[6] G. Stober and J. L. Chau, "A multistatic and multifrequency novel approach for specular meteor radars to improve wind measurements in the MLT region," Radio Science, vol. 50, no. 5, pp. 431-442, 2015, 2014RS005591. [Online]. Available: http://dx.doi.org/10.1002/2014RS005591

[7] J. Vierinen, J. L. Chau, N. Pfeffer, M. Clahsen, and G. Stober, "Coded continuous wave meteor radar," Atmospheric Measurement Techniques, vol. 9, no. 2, pp. 829-839, 2016. [Online]. Available: https://www.atmos-meas-tech.net/9/829/2016/

[8] J. L. Chau, J. M. Urco, J. P. Vierinen, R. A. Volz, M. Clahsen, N. Pfeffer, and J. Trautner, "Novel specular meteor radar systems using coherent mimo techniques to study the mesosphere and lower thermosphere," Atmospheric Measurement Techniques, vol. 12, no. 4, pp. 2113-2127, 2019. [Online]. Available: https://www.atmos-meastech.net/12/2113/2019/

[9] J. M. Urco, J. L. Chau, M. A. Milla, J. P. Vierinen, and T. Weber, "Coherent MIMO to improve aperture synthesis radar imaging of fieldaligned irregularities: First results at Jicamarca," IEEE Transactions on Geoscience and Remote Sensing, vol. PP, no. 99, pp. 1-11, 2018.

[10] C. Wang, E. K. S. Au, R. D. Murch, W. H. Mow, R. S. Cheng, and $\mathrm{V}$. Lau, "On the performance of the MIMO zero-forcing receiver in the presence of channel estimation error," IEEE Transactions on Wireless Communications, vol. 6, no. 3, pp. 805-810, March 2007.

[11] M. L. Ammari and P. Fortier, "Analysis of MIMO receiver using generalized least squares method in colored environments," Journal of Computer Networks and Communications, vol. 2014, 2014.

[12] R. Radzokota, E. Golovins, and N. Ventura, "Linear detector performance in ill-conditioned MIMO OFDM channel," in 2009 International Conference on Ultra Modern Telecommunications Workshops, Oct 2009, pp. 1-7.
[13] G. Turin, "An introduction to matched filters," IRE Transactions on Information Theory, vol. 6, no. 3, pp. 311-329, June 1960

[14] J. Jones, A. R. Webster, and W. K. Hocking, "An improved interferometer design for use with meteor radars," Radio Science, vol. 33, no. 1, pp. 55-65, 1998. [Online]. Available: https://agupubs.onlinelibrary.wiley.com/doi/abs/10.1029/97RS03050

[15] P. C. Haneen, "The truncated SVD as a method for regularization," BIT Numerical Mathematics, vol. 27, no. 4, pp. 534-553, Dec 1987. [Online]. Available: https://doi.org/10.1007/BF01937276

[16] P. C. Hansen, "Truncated singular value decomposition solutions to discrete ill-posed problems with ill-determined numerical rank," SIAM Journal on Scientific and Statistical Computing, vol. 11, no. 3, pp. 503-518, 1990. [Online]. Available: https://doi.org/10.1137/0911028

[17] A. E. Hoerl and R. W. Kennard, "Ridge regression: Biased estimation for nonorthogonal problems," Technometrics, vol. 12, no. 1, pp. 55-67, 1970.

[18] G. James, D. Witten, T. Hastie, and R. Tibshirani, An introduction to statistical learning. Springer, 2009, vol. 7.

[19] R. J. Tibshirani and R. Tibshirani, "A bias correction for the minimum error rate in cross-validation," Ann. Appl. Stat., vol. 3, no. 2, pp. 822829, 06 2009. [Online]. Available: https://doi.org/10.1214/08-AOAS224

[20] S. M. Kay, Fundamentals of Statistical Signal Processing: Estimation Theory. Upper Saddle River, NJ, USA: Prentice-Hall, Inc., 1993.

[21] P. W. Wolniansky, G. J. Foschini, G. D. Golden, and R. A. Valenzuela, "V-BLAST: an architecture for realizing very high data rates over the rich-scattering wireless channel," in 1998 URSI International Symposium on Signals, Systems, and Electronics. Conference Proceedings (Cat. No.98EX167), Oct 1998, pp. 295-300.

[22] G. Wang, D. Wang, and D. Li, "An efficient ZF-SIC detection algorithm in MIMO CDMA system," in 14th IEEE Proceedings on Personal, Indoor and Mobile Radio Communications, 2003. PIMRC 2003., vol. 2, Sep. 2003, pp. 1708-1711 vol.2.

[23] J. A. Högbom, "Aperture synthesis with a non-regular distribution of interferometer baselines," Astronomy and Astrophysics Supplement, vol. 15, p. 417, Jun. 1974.

[24] T. T. Cai and L. Wang, "Orthogonal matching pursuit for sparse signal recovery with noise," IEEE Transactions on Information Theory, vol. 57, no. 7, pp. 4680-4688, July 2011.

[25] D. L. Donoho, "Compressed sensing," IEEE Transactions on Information Theory, vol. 52, no. 4, pp. 1289-1306, April 2006.

[26] E. J. Cands, J. K. Romberg, and T. Tao, "Stable signal recovery from incomplete and inaccurate measurements," Communications on Pure and Applied Mathematics, vol. 59, no. 8, pp. 1207-1223, 2006. [Online]. Available: https://onlinelibrary.wiley.com/doi/abs/10.1002/cpa.20124

[27] E. J. Candes and T. Tao, "Decoding by linear programming," IEEE Transactions on Information Theory, vol. 51, no. 12, pp. 4203-4215, Dec 2005.

[28] E. J. Cands, J. Romberg, and T. Tao, "Robust uncertainty principles: exact signal reconstruction from highly incomplete frequency information," IEEE Transactions on Information Theory, vol. 52, no. 2, pp. 489-509, Feb 2006.

[29] W. Dan and R. Wang, "Robustness of orthogonal matching pursuit under restricted isometry property," Science China Mathematics, vol. 57, no. 3, pp. 627-634, Mar 2014. [Online]. Available: https://doi.org/10.1007/s11425-013-4655-4

[30] Y. Shen and S. Li, "Sparse signals recovery from noisy measurements by orthogonal matching pursuit," Inverse Problems \& Imaging, vol. 9, p. $231,2015$.

[31] T. Wimalajeewa, Y. C. Eldar, and P. K. Varshney, "Recovery of sparse matrices via matrix sketching," CoRR, vol. abs/1311.2448, 2013. [Online]. Available: http://arxiv.org/abs/1311.2448

[32] J. A. Tropp, "Greed is good: algorithmic results for sparse approximation," IEEE Transactions on Information Theory, vol. 50, no. 10, pp. 2231-2242, Oct 2004.

[33] H. Bi, C. Zhao, H. Bi, Y. Liu, and N. Li, "Performance evaluation of greedy reconstruction algorithms in compressed sensing," in 2016 9th International Congress on Image and Signal Processing, BioMedical Engineering and Informatics (CISP-BMEI), Oct 2016, pp. 1322-1327.

[34] D. L. Donoho, Y. Tsaig, I. Drori, and J. Starck, "Sparse solution of underdetermined systems of linear equations by stagewise orthogonal matching pursuit," IEEE Transactions on Information Theory, vol. 58, no. 2, pp. 1094-1121, Feb 2012.

[35] J. Wang, S. Kwon, and B. Shim, "Generalized orthogonal matching pursuit," IEEE Transactions on Signal Processing, vol. 60, no. 12, pp. 6202-6216, Dec 2012. 
[36] D. Needell and J. Tropp, "CoSaMP: Iterative signal recovery from incomplete and inaccurate samples," Applied and Computational Harmonic Analysis, vol. 26, no. 3, pp. 301 - 321, 2009. [Online]. Available: http://www.sciencedirect.com/science/article/pii/S1063520308000638

[37] D. Needell and R. Vershynin, "Signal recovery from incomplete and inaccurate measurements via regularized orthogonal matching pursuit," IEEE Journal of Selected Topics in Signal Processing, vol. 4, no. 2, pp. 310-316, April 2010.

[38] Y. C. Eldar, P. Kuppinger, and H. Bolcskei, "Block-sparse signals: Uncertainty relations and efficient recovery," IEEE Transactions on Signal Processing, vol. 58, no. 6, pp. 3042-3054, June 2010.

[39] V. N. Xuan, K. Hartmann, W. Weihs, and O. Loffeld, "Modified orthogonal matching pursuit for multiple measurement vector with joint sparsity in super-resolution compressed sensing," in 2017 51st Asilomar Conference on Signals, Systems, and Computers, Oct 2017, pp. 840-844.

[40] E. J. Candes and T. Tao, "Near-optimal signal recovery from random projections: Universal encoding strategies?" IEEE Transactions on Information Theory, vol. 52, no. 12, pp. 5406-5425, Dec 2006.

[41] D. L. Donoho and X. Huo, "Uncertainty principles and ideal atomic decomposition," IEEE Trans. Inf. Theor, vol. 47, no. 7, pp. 2845-2862, Sep. 2006. [Online]. Available: http://dx.doi.org/10.1109/18.959265

[42] S. D. Blunt and E. L. Mokole, "Overview of radar waveform diversity," IEEE Aerospace and Electronic Systems Magazine, vol. 31, no. 11, pp. 2-42, November 2016.

[43] R. Gold, "Optimal binary sequences for spread spectrum multiplexing (corresp.)," IEEE Transactions on Information Theory, vol. 13, no. 4, pp. 619-621, October 1967.

[44] R. Frank, "Polyphase codes with good nonperiodic correlation properties," IEEE Trans. Inf. Theor., vol. 9, no. 1, pp. 43-45, Sep. 2006. [Online]. Available: http://dx.doi.org/10.1109/TIT.1963.1057798

[45] Intel, "Intel distribution for Python." [Online]. Available: https://software.intel.com/en-us/distribution-for-python

[46] G. Golub and C. van Loan, "An analysis of the total least squares problem," SIAM Journal on Numerical Analysis, vol. 17, no. 6, pp. 883-893, 1980. [Online]. Available: https://doi.org/10.1137/0717073

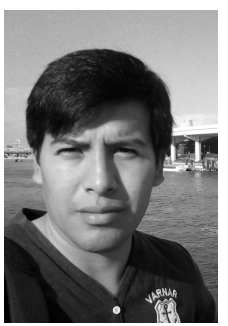

Juan M. Urco received the B.S. degree in electrical engineering from the Universidad Nacional Mayor de San Marcos, Lima, Perú in 2006. From 2008 to 2015, he worked as a signal-processing engineer at the Jicamarca Radio Observatory and as a hardware design engineer in the private sector. In 2016, he started his graduate studies joining the Radar Remote Sensing department at the Leibniz Institute of Atmospheric Physics in Kühlungsborn, Germany. His main area of research is in developing and applying new remote sensing techniques for atmospheric and ionospheric observations. He is currently pursuing the Ph.D. degree in electrical engineering at University of Rostock, Germany.

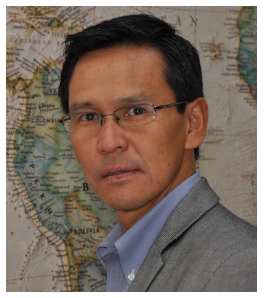

Jorge L. Chau is Professor of Atmospheric Physics at the University of Rostock and Head of the Radar Remote Sensing department at the Leibniz Institute of Atmospheric Physics in Kühlungsborn, Germany. He received his M.S. in 1995 and Ph.D. in 1998 in Electrical and Computer Engineering from the University of Colorado at Boulder, USA. From 2001 to 2012, he served as Director of the Jicamarca Radio Observatory in Lima, Peru. His research has focused on radar and radio studies of the neutral upper atmosphere and ionosphere. He is interested in the development and implementation of radar techniques to improve the atmospheric and ionospheric measurements as well as to improve the understanding of the atmospheric and ionospheric physics at low, mid and high latitudes.

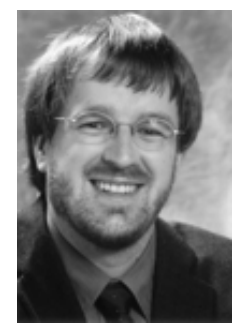

Tobias Weber (M'00-SM'14) received the Dipl.Ing. degree in electrical engineering, and the Ph.D. and Habilitation degrees from the University of Kaiserslautern, Kaiserslautern, Germany, in 1996, 1999, and 2003, respectively. From 1996 to 2005, he was a member of the staff of the Research Group for RF Communications, University of Kaiserslautern. From 1996 to 1999 , he was active in the development of a hardware demonstrator for a $3 \mathrm{rd}$ generation mobile radio system, where his work focused on future signal processing concepts. In 2005, he became a professor of microwave technology with the University of Rostock, Rostock, Germany. His research interests include future mobile radio systems, OFDM mobile radio systems, MIMO techniques, and localization techniques. He is a member of Verband Deutscher ElektrotechnikerInformationstechnische Gesellschaft (VDE/ITG).

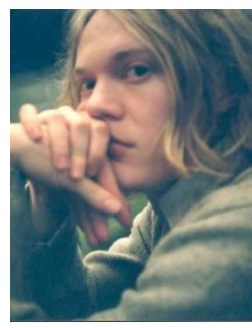

Juha P. Vierinen is an Associate Professor of Space Physics at University of Troms $\varnothing$. He received a M.S. in 2005 and Ph.D. in 2012 from Helsinki University of Technology. From 2013 to 2016, he worked at the MIT Haystack Observatory. He is interested in the development of novel radar and radio remote sensing measurement techniques to study space plasmas.

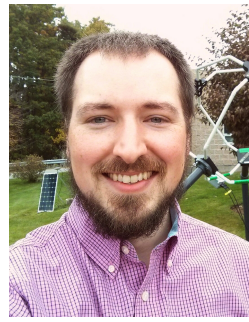

Ryan Volz is a Research Scientist at MIT Haystack Observatory with interests in signal processing, statistical estimation, and novel instrumentation applied particularly to radio science. He earned a BS degree in Aerospace Engineering from the Pennsylvania State University in 2007, an M.Phil degree in Engineering (Control Systems) from the University of Cambridge in 2008, and MS and $\mathrm{PhD}$ degrees in Aeronautics and Astronautics from Stanford University in 2009 and 2015, respectively. He was awarded an NDSEG graduate fellowship in 2008 and received third prize at the international URSI GASS 2011 student paper competition for his work on sparsity-based inversion of radar signals. 